\title{
On Bending of Bernoulli-Euler Nanobeams for Nonlocal Composite Materials
}

\author{
Luciano Feo and Rosa Penna \\ Department of Civil Engineering, University of Salerno, Via Giovanni Paolo II, 132, 84084 Fisciano, Italy \\ Correspondence should be addressed to Luciano Feo; 1.feo@unisa.it
}

Received 4 December 2015; Accepted 24 April 2016

Academic Editor: Theodoros C. Rousakis

Copyright (C) 2016 L. Feo and R. Penna. This is an open access article distributed under the Creative Commons Attribution License, which permits unrestricted use, distribution, and reproduction in any medium, provided the original work is properly cited.

\begin{abstract}
Evaluation of size effects in functionally graded elastic nanobeams is carried out by making recourse to the nonlocal continuum mechanics. The Bernoulli-Euler kinematic assumption and the Eringen nonlocal constitutive law are assumed in the formulation of the elastic equilibrium problem. An innovative methodology, characterized by a lowering in the order of governing differential equation, is adopted in the present manuscript in order to solve the boundary value problem of a nanobeam under flexure. Unlike standard treatments, a second-order differential equation of nonlocal equilibrium elastic is integrated in terms of transverse displacements and equilibrated bending moments. Benchmark examples are developed, thus providing the nonlocality effect in nanocantilever and clampled-simply supported nanobeams for selected values of the Eringen scale parameter.
\end{abstract}

\section{Introduction}

Analysis of nanodevices is a subject of special interest in the current literature. Particular attention is given to the static behavior of beam-like components of nanoelectromechanical systems (NEMS). Nonlocal constitutive behaviors are adequate in order to evaluate the size phenomenon in nanostructures; see, for example, [1-9]. Investigations on random elastic structures have been carried out in [10-13]. Many research efforts have been devoted to theoretical and computational advances about specific structural models [14-19]. Recent variational formulations of nonlocal continua have been developed in [20-22]. Noteworthy theoretical results on functionally graded nanobeams have been contributed in [23-25]. Nevertheless, exact solutions are not always available so that finite element strategies are needful; see, for example, [26]. Micromechanical approaches are broadly used in order to analyze the effective behavior of composite structures [27].

Innovative applications of engineering interest are proposed in [28-30]. Numerical and experimental methodologies for composite structures are developed in [31, 32]. Effective applications of tensionless models concerning crack propagation are reported in [33-37]. A skillful analysis of equilibrium configurations of hyperelastic cylindrical bodies and compressible cubes is carried out in [38].
The present paper deals with one-dimensional nanostructure by making recourse to the tools of nonlocal continuum mechanics. Small-scale effects exhibited by functionally graded nanobeams under flexure are analyzed in Section 2.

\section{Nonlocal Elasticity}

In local linear elasticity for isotropic materials, stress and strain at a point $\mathbf{x}$ of a Cauchy continuum are functionally related by the following classical law:

$$
\boldsymbol{\sigma}_{i j}(\mathbf{x})=2 \mu \mathbf{e}_{i j}(\mathbf{x})+\lambda \mathbf{e}_{r r}(\mathbf{x}) \delta_{i j},
$$

with $\mu$ and $\lambda$ LAMÉ constants.

Such a constitutive behavior is not adequate to evaluate size effects in nanostructures. An effective law able to capture scale phenomena was developed by Eringen in [39] who defined the following nonlocal integral operator:

$$
\mathbf{t}_{i j}(\mathbf{x})=\int_{V} K\left(\left|\mathbf{x}^{\prime}-\mathbf{x}\right|, \tau\right) \boldsymbol{\sigma}_{i j}\left(\mathbf{x}^{\prime}\right) d V,
$$

where

(1) $\mathbf{t}_{i j}$ is the nonlocal stress,

(2) $\boldsymbol{\sigma}_{i j}$ is the macroscopic stress given by (5), 
(3) $K$ is the influence function,

(4) $\tau=e_{0} a / l$ a dimensionless nonlocal parameter defined in terms of the material constant $e_{0}$ and of the internal and external characteristic lengths $a$ and $l$, respectively.

In agreement with the Eringen proposal in choosing the following influence function $1-c^{2} \nabla^{2}$, the nonlocal elastic law (2) rewrites as

$$
\left(1-\left(e_{0} a\right)^{2} \nabla^{2}\right) \mathbf{t}_{i j}=\boldsymbol{\sigma}_{i j}
$$

where $\nabla^{2}$ denotes the Laplace operator. The differential form adopted for bending of nanobeams, analogous to (3), is provided by

$$
\bar{\sigma}-c^{2} \frac{d^{2} \bar{\sigma}}{d x^{2}}=\sigma
$$

where $\bar{\sigma}$ is the nonlocal normal stress and $\sigma$ is the macroscopic normal stress on cross sections. Note that the stress $\sigma$ is expressed in terms of elastic axial strains by

$$
\sigma=E \varepsilon,
$$

with $E$ Young modulus.

\section{Bending of Nonlocal Nanobeams}

Let us consider a bent nanobeam of length $L$, with Young modulus $E$ functionally graded in the cross section $\Omega$ and uniform along the beam axis $x$. The cross-sectional elastic centre and the principal axes of elastic inertia, associated with the scalar field $E$, are, respectively, denoted by $\mathbf{G}$ and by the pair $(y, z)$.

The nanobeam is assumed to be subjected in the plane $(x, y)$ to the following loading conditions:

$q_{t}$, distributed load per unit length in the interval $[0, L]$,

$\mathscr{F}_{t}$, concentrated forces at the end cross sections $\{0, L\}$,

$\mathscr{M}$, concentrated couples at the end cross sections $\{0, L\}$.

The bending stiffness is defined by

$$
k_{b}:=\int_{\Omega} E y^{2} d A
$$

Differential and boundary conditions of equilibrium are expressed by

$$
\begin{aligned}
& \frac{d^{2} M_{b}}{d x^{2}}=q_{t}, \quad \text { in }[0, L], \\
& \frac{d M_{b}}{d x}= \pm \mathscr{F}_{t}, \quad \text { at }\{0, L\}, \\
& M_{b}=\mp \mathscr{M}, \quad \text { at }\{0, L\},
\end{aligned}
$$

where $M_{b}$ is the bending moment.
The bending curvature, corresponding to the transverse displacement $v$, is given by

$$
\chi(x)=\frac{d^{2} v}{d x^{2}}(x)
$$

The differential equation of nonlocal elastic equilibrium of a nanobeam under flexure is formulated as follows. Let us preliminarily multiply (4) by the coordinate $y$ along the bending axis and integrate on the cross section $\Omega$ :

$$
\int_{\Omega} \bar{\sigma} y d A-\left(e_{0} a\right)^{2} \int_{\Omega} \frac{d^{2} \bar{\sigma}}{d x^{2}} y d A=\int_{\Omega} E \mathcal{E} y d A,
$$

with the axial dilation provided by the known formula $\varepsilon(x)=$ $-\chi(x) y$.

Enforcing (8) and (7) $)_{1}$ and imposing the static equivalence condition

$$
M_{b}=-\int_{\Omega} \sigma y d A,
$$

we obtain the relation

$$
M_{b}-\left(e_{0} a\right)^{2} q_{t}=k_{b} \frac{d^{2} v}{d x^{2}} .
$$

This equation can be interpreted as decomposition formula of the bending curvature $\chi_{b}=d^{2} v / d x^{2}$ into elastic $\chi_{\mathrm{EL}}$ and inelastic $\chi_{\text {IN }}$ parts

$$
\chi_{b}=\chi_{\mathrm{EL}}+\chi_{\mathrm{IN}}
$$

with

$$
\begin{gathered}
\chi_{\mathrm{EL}}=\frac{M_{b}}{k_{b}}, \\
\chi_{\mathrm{IN}}=-\frac{\left(e_{0} a\right)^{2}}{k_{b}} q_{t} .
\end{gathered}
$$

Accordingly, the scale effect exhibited by bending moments and displacements of a FG nonlocal nanobeam can be evaluated by solving a corresponding linearly elastic beam subjected to the bending curvature distortion $\chi_{\mathrm{IN}}(13)_{2}$.

\section{Examples}

The solution methodology of the nonlocal elastic equilibrium problem of a nanobeam enlightened in the previous section is here adopted in order to assess small-scale effects in nanocantilever and clamped-simply supported nanobeams under a uniformly distributed load $q_{t}$. The nonlocality effect on the transverse displacement is thus due to the uniform bending curvature distortion formulated in (13) 2 . Graphical evidences of the elastic displacements are provided in Figures 1 and 2, in terms of the following dimensionless parameters $\xi=x / L$ and $v^{*}(\xi)=\left(100 k_{b}\right) /\left(q_{t} L^{4}\right) v(\xi)$, for selected values of the nonlocal parameter $\tau:=e_{0} a / L$. Details of the calculations and some comments are reported below.

4.1. Cantilever Nanobeam. The bending moment is given by

$$
M_{b}(x)=\frac{q_{t}}{2} x^{2}-q_{t} L x+\frac{q_{t} L^{2}}{2} .
$$




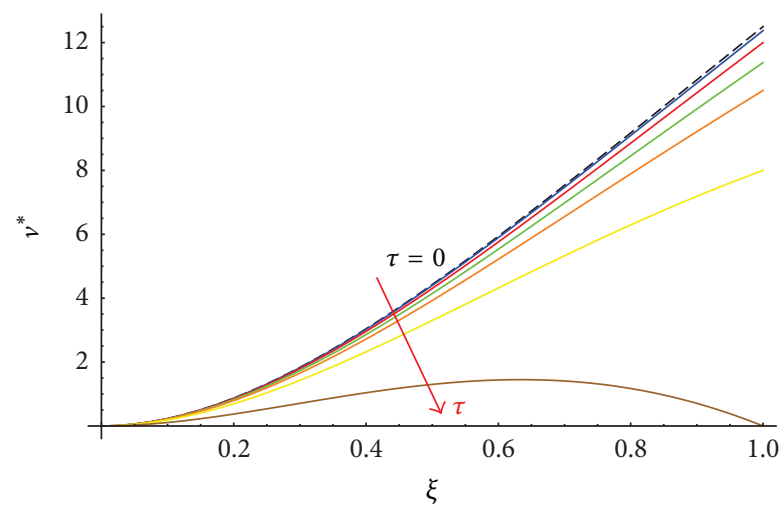

Figure 1: Dimensionless transverse displacements $v^{*}$ for the nanocantilever versus $\xi$ for $\tau \in\{0,0.05,0.1,0.15,0.2,0.3,0.5\}$.

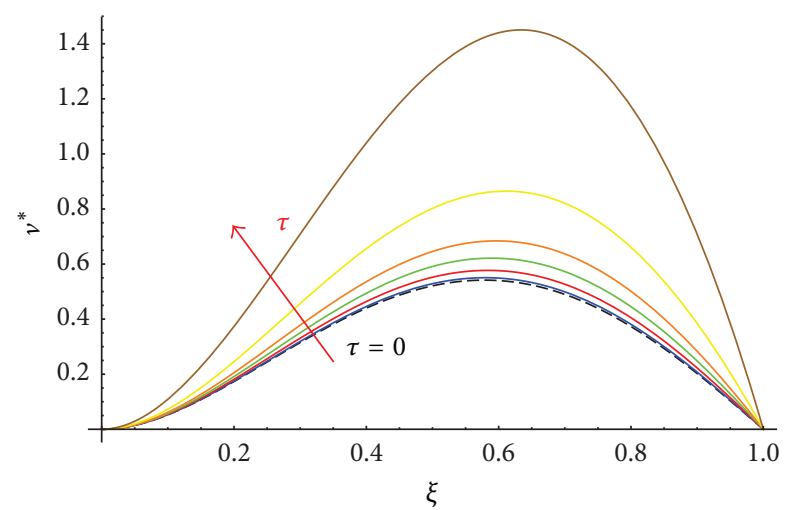

Figure 2: Dimensionless transverse displacement $v^{*}$ for the clamped-simply supported nanobeam versus $\xi$ for $\tau \in\{0,0.05,0.1$, $0.15,0.2,0.3,0.5\}$.

The 1.h.s. of (11) is hence known, so that the differential condition of nonlocal elastic equilibrium to be integrated writes explicitly as

$$
k_{b} \frac{d^{2} v}{d x^{2}}(x)=M_{b}(x)-\tau^{2} L^{2} q_{t}
$$

The general integral of (15) takes the form

$$
v(x)=a x+b+\bar{v}(x)
$$

where

$$
\bar{v}(x)=-\frac{q_{t}}{24 k_{b}} x^{4}+\frac{q_{t} L}{6 k_{b}} x^{3}-\frac{q_{t} L^{2}}{4} x^{2}-\frac{\tau^{2} L^{2} q_{t}}{2 k_{b}} x^{2}
$$

is a particular solution of (15). The evaluation of the integration constants $a$ and $b$ is carried out by prescribing the boundary conditions

$$
\begin{gathered}
v(0)=0, \\
\frac{d v}{d x}(0)=0 .
\end{gathered}
$$

The transversal deflection follows by a direct computation

$$
v(x)=\frac{q_{t}}{24 k_{b}} x^{4}-\frac{q_{t} L}{6 k_{b}} x^{3}+\frac{q_{t} L^{2}}{4 k_{b}} x^{2}-\frac{\tau^{2} q_{t} L^{2}}{2 k_{b}} x^{2} .
$$

The maximum displacement is given by

$$
v_{m}=v(L)=\left(\frac{1}{8}-\frac{\tau^{2}}{2}\right) \frac{q_{t} L^{4}}{k_{b}} .
$$

Nanocantilever becomes stiffer with increasing the nonlocal parameter $\tau$. Indeed, according to the analysis proposed in Section 3 , the sign of the prescribed distortion $\chi_{\mathrm{IN}}$, describing the nonlocality effect, is opposite to the one of the elastic curvature $\chi_{\mathrm{EL}}$. In particular, the displacement of the free end vanishes if $\tau=0.5$ (see Figure 1) according to (20).

It is worth noting that, with the structure being statically determinate, the bending moment (14) is not affected by the scale phenomenon.

4.2. Clamped-Simply Supported Nanobeam. Equilibrated bending moments are provided by the relation

$$
M_{b}(x)=a_{1}(x-L)+\frac{q_{t} L^{2}}{2}-\frac{q_{t}}{2} x^{2}
$$

with $a_{1} \in \mathscr{R}$ being an arbitrary constant. The differential condition of nonlocal elastic equilibrium (11) to be integrated takes thus the form

$$
-k_{b} \frac{d^{2} v}{d x^{2}}(x)=a_{1}(x-L)+\frac{q_{t} L^{2}}{2}-\frac{q_{t}}{2} x^{2}+\tau^{2} L^{2} q_{t} .
$$

Equation (22) is solved by imposing the following kinematic boundary conditions:

$$
\begin{gathered}
v(0)=0, \\
\frac{d v}{d x}(0)=0, \\
v(L)=0 .
\end{gathered}
$$

A direct computation gives the transversal displacement field

$$
v(x)=\frac{q_{t}}{24 k_{b}} x^{4}-\frac{5 q_{t} L}{48 k_{b}} x^{3}+\frac{q_{t} L^{2}}{16 k_{b}} x^{2}+\frac{\tau^{2} q_{t} L}{4 k_{b}} x^{2}(L-x)
$$

and the bending moment

$$
M_{b}(x)=\frac{q_{t}}{2} x^{2}-\frac{q_{t} L}{2}\left(\frac{5}{4}+3 \tau^{2}\right) x+\frac{q_{t} L^{2}}{2}\left(3 \tau^{2}+\frac{1}{4}\right) .
$$

The maximum displacement is given by

$$
v_{m}=v(\bar{x}),
$$

with $\bar{x}=3 L\left((3 / 2)\left(5 / 24+\tau^{2} / 2\right)-\sqrt{(9 / 16) \tau^{4}+11 / 768+(13 / 96) \tau^{2}}\right)$ solution of the equation (the known local maximum point $\bar{x} \simeq 0.578 L$ is recovered by setting $\tau=0)(d v / d x)(x)=0$.

In agreement with the equivalence method exposed in Section 3, (24) and (25) provide the deflection and 


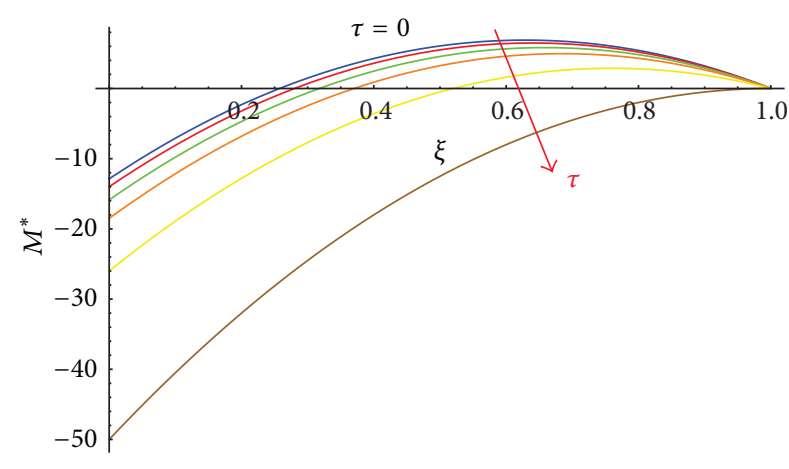

FIgURE 3: Dimensionless bending moment $M^{*}$ versus dimensionless abscissa $\xi$ of a clamped-simply supported nanobeam.

bending moment of a corresponding local nanobeam under the transversal load distribution $q_{t}$ and the distortion $\chi_{\mathrm{IN}}$ equivalent to the nonlocality effect.

A plot of the dimensionless bending moment $M^{*}=$ $-\left(100 / q_{t} L^{2}\right) M$ versus the dimensionless parameter $\xi=x / L$ is given in Figure 3 for increasing values of the nonlocal parameter $\tau$.

\section{Conclusion}

The Eringen nonlocal law has been used in order to assess size effects in nanobeams formulated according to the BernoulliEuler kinematics. The treatment extends to functionally graded materials the analysis carried out in [24] under the special assumption of elastically homogeneous nanobeams. Transverse deflections of cantilever and clamped-simply supported nanobeams have been established for different values of the nonlocal parameter. Such analytical solutions could be conveniently adopted by other scholars as simple reference examples for numerical evaluations in nonlocal composite mechanics.

\section{Competing Interests}

The authors declare that they have no competing interests.

\section{References}

[1] R. de Borst and H.-B. Muehlhaus, "Gradient-dependent plasticity: formulation and algorithmic aspects," International Journal for Numerical Methods in Engineering, vol. 35, no. 3, pp. 521-539, 1992.

[2] E. C. Aifantis, "Gradient deformation models at nano, micro, and macro scales," Journal of Engineering Materials and Technology, vol. 121, pp. 189-202, 1999.

[3] B. Arash and Q. Wang, "A review on the application of nonlocal elastic models in modeling of carbon nanotubes and graphenes," Computational Materials Science, vol. 51, no. 1, pp. 303-313, 2012.

[4] R. Rafiee and R. M. Moghadam, "On the modeling of carbon nanotubes: a critical review," Composites Part B: Engineering, vol. 56, pp. 435-449, 2014.
[5] J. N. Reddy, "Nonlocal theories for bending, buckling and vibration of beams," International Journal of Engineering Science, vol. 45 , no. 2-8, pp. 288-307, 2007.

[6] Ö. Civalek and Ç. Demir, "Bending analysis of microtubules using nonlocal Euler-Bernoulli beam theory," Applied Mathematical Modelling, vol. 35, no. 5, pp. 2053-2067, 2011.

[7] H.-T. Thai and T. P. Vo, "A nonlocal sinusoidal shear deformation beam theory with application to bending, buckling, and vibration of nanobeams," International Journal of Engineering Science, vol. 54, pp. 58-66, 2012.

[8] Q. Wang and K. M. Liew, "Application of nonlocal continuum mechanics to static analysis of micro- and nano-structures," Physics Letters A, vol. 363, no. 3, pp. 236-242, 2007.

[9] R. Barretta, L. Feo, R. Luciano, and F. Marotti de Sciarra, "Application of an enhanced version of the Eringen differential model to nanotechnology," Composites Part B: Engineering, vol. 96, pp. 274-280, 2016.

[10] R. Luciano and J. R. Willis, "Bounds on non-local effective relations for random composites loaded by configurationdependent body force," Journal of the Mechanics and Physics of Solids, vol. 48, no. 9, pp. 1827-1849, 2000.

[11] R. Luciano and J. R. Willis, "Non-local constitutive response of a random laminate subjected to configuration-dependent body force," Journal of the Mechanics and Physics of Solids, vol. 49, no. 2, pp. 431-444, 2001.

[12] R. Luciano and J. R. Willis, "Boundary-layer corrections for stress and strain fields in randomly heterogeneous materials," Journal of the Mechanics and Physics of Solids, vol. 51, no. 6, pp. 1075-1088, 2003.

[13] R. Luciano and J. R. Willis, "Hashin-Shtrikman based FE analysis of the elastic behaviour of finite random composite bodies," International Journal of Fracture, vol. 137, no. 1-4, pp. 261-273, 2006.

[14] A. Caporale, R. Luciano, and L. Rosati, "Limit analysis of masonry arches with externally bonded FRP reinforcements," Computer Methods in Applied Mechanics and Engineering, vol. 196, no. 1-3, pp. 247-260, 2006.

[15] F. Marmo and L. Rosati, "Analytical integration of elasto-plastic uniaxial constitutive laws over arbitrary sections," International Journal for Numerical Methods in Engineering, vol. 91, no. 9, pp. 990-1022, 2012.

[16] F. Marmo and L. Rosati, "The fiber-free approach in the evaluation of the tangent stiffness matrix for elastoplastic uniaxial constitutive laws," International Journal for Numerical Methods in Engineering, vol. 94, no. 9, pp. 868-894, 2013.

[17] R. Barretta, "Analogies between Kirchhoff plates and SaintVenant beams under flexure," Acta Mechanica, vol. 225, no. 7, pp. 2075-2083, 2014.

[18] L. Rosati and F. Marmo, "Closed-form expressions of the thermo-mechanical fields induced by a uniform heat source acting over an isotropic half-space," International Journal of Heat and Mass Transfer, vol. 75, pp. 272-283, 2014.

[19] F. Marmo and L. Rosati, "A general approach to the solution of boussinesq's problem for polynomial pressures acting over polygonal domains," Journal of Elasticity, vol. 122, no. 1, pp. 75112, 2016.

[20] F. Marotti de Sciarra and M. Salerno, "On thermodynamic functions in thermoelasticity without energy dissipation," European Journal of Mechanics-A/Solids, vol. 46, pp. 84-95, 2014.

[21] F. Marotti de Sciarra, "Finite element modelling of nonlocal beams," Physica E, vol. 59, pp. 144-149, 2014. 
[22] F. Marotti de Sciarra and R. Barretta, "A new nonlocal bending model for Euler-Bernoulli nanobeams," Mechanics Research Communications, vol. 62, no. 1, pp. 25-30, 2014.

[23] R. Barretta, R. Luciano, and F. Marotti de Sciarra, "A fully gradient model for Euler-Bernoulli nanobeams," Mathematical Problems in Engineering, vol. 2015, Article ID 495095, 8 pages, 2015.

[24] R. Barretta and F. Marotti de Sciarra, "Analogies between nonlocal and local Bernoulli-Euler nanobeams," Archive of Applied Mechanics, vol. 85, no. 1, pp. 89-99, 2015.

[25] R. Barretta, L. Feo, R. Luciano, F. Marotti de Sciarra, and R. Penna, "Functionally graded Timoshenko nanobeams: a novel nonlocal gradient formulation," Composites Part B: Engineering, vol. 100, pp. 208-219, 2016.

[26] F. Greco and R. Luciano, "A theoretical and numerical stability analysis for composite micro-structures by using homogenization theory," Composites Part B: Engineering, vol. 42, no. 3, pp. 382-401, 2011.

[27] A. Caporale, L. Feo, D. Hui, and R. Luciano, "Debonding of FRP in multi-span masonry arch structures via limit analysis," Composite Structures, vol. 108, no. 1, pp. 856-865, 2014.

[28] V. A. Salomoni, C. Majorana, G. M. Giannuzzi, and A. Miliozzi, "Thermal-fluid flow within innovative heat storage concrete systems for solar power plants," International Journal of Numerical Methods for Heat \& Fluid Flow, vol. 18, pp. 969-999, 2008.

[29] V. A. Salomoni, G. Mazzucco, C. Pellegrino, and C. E. Majorana, "Three-dimensional modelling of bond behaviour between concrete and FRP reinforcement," Engineering Computations, vol. 28, no. 1, pp. 5-29, 2011.

[30] V. A. Salomoni, C. E. Majorana, B. Pomaro, G. Xotta, and F. Gramegna, "Macroscale and mesoscale analysis of concrete as a multiphase material for biological shields against nuclear radiation," International Journal for Numerical and Analytical Methods in Geomechanics, vol. 38, no. 5, pp. 518-535, 2014.

[31] G. Dinelli, G. Belz, C. E. Majorana, and B. A. Schrefler, "Experimental investigation on the use of fly ash for lightweight precast structural elements," Materials and Structures, vol. 29, no. 194, pp. 632-638, 1996.

[32] G. Xotta, G. Mazzucco, V. A. Salomoni, C. E. Majorana, and K. J. Willam, "Composite behavior of concrete materials under high temperatures," International Journal of Solids and Structures, vol. 64, pp. 86-99, 2015.

[33] A. M. Tarantino, "Nonlinear fracture mechanics for an elastic Bell material," The Quarterly Journal of Mechanics \& Applied Mathematics, vol. 50, part 3, pp. 435-456, 1997.

[34] A. M. Tarantino, "The singular equilibrium field at the notchtip of a compressible material in finite elastostatics," Journal of Applied Mathematics and Physics, vol. 48, no. 3, pp. 370-388, 1997.

[35] A. M. Tarantino, "On extreme thinning at the notch-tip of a neoHookean sheet," The Quarterly Journal of Mechanics \& Applied Mathematics, vol. 51, part 2, pp. 179-190, 1998.

[36] A. M. Tarantino, "On the finite motions generated by a mode I propagating crack," Journal of Elasticity, vol. 57, no. 2, pp. 85$103,1999$.

[37] A. M. Tarantino, "Crack propagation in finite elastodynamics," Mathematics and Mechanics of Solids, vol. 10, no. 6, pp. 577-601, 2005.

[38] A. M. Tarantino, "Homogeneous equilibrium configurations of a hyperelastic compressible cube under equitriaxial dead-load tractions," Journal of Elasticity, vol. 92, no. 3, pp. 227-254, 2008.
[39] A. C. Eringen, "On differential equations of nonlocal elasticity and solutions of screw dislocation and surface waves," Journal of Applied Physics, vol. 54, no. 9, pp. 4703-4710, 1983. 


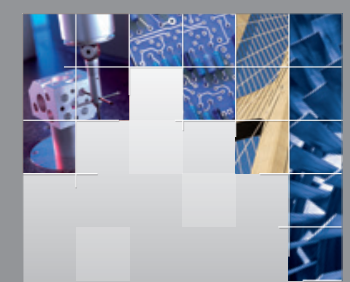

\section{Enfincering}
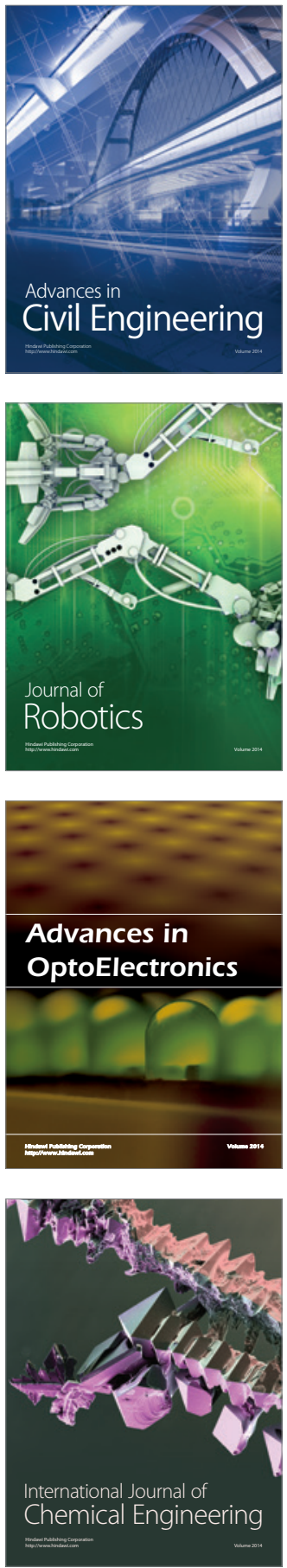

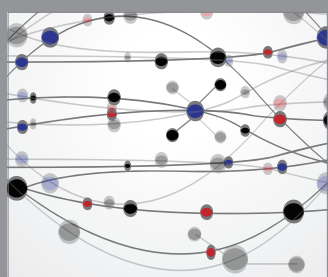

The Scientific World Journal

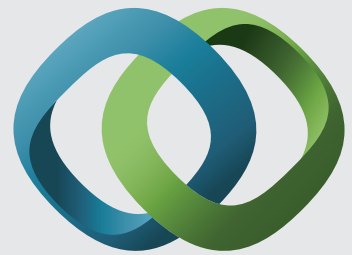

\section{Hindawi}

Submit your manuscripts at

http://www.hindawi.com
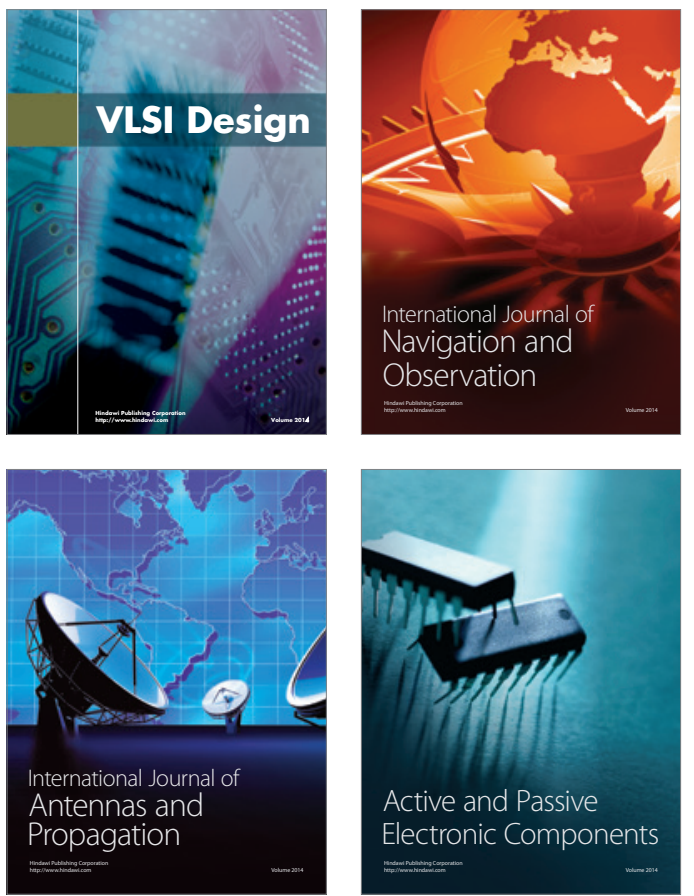
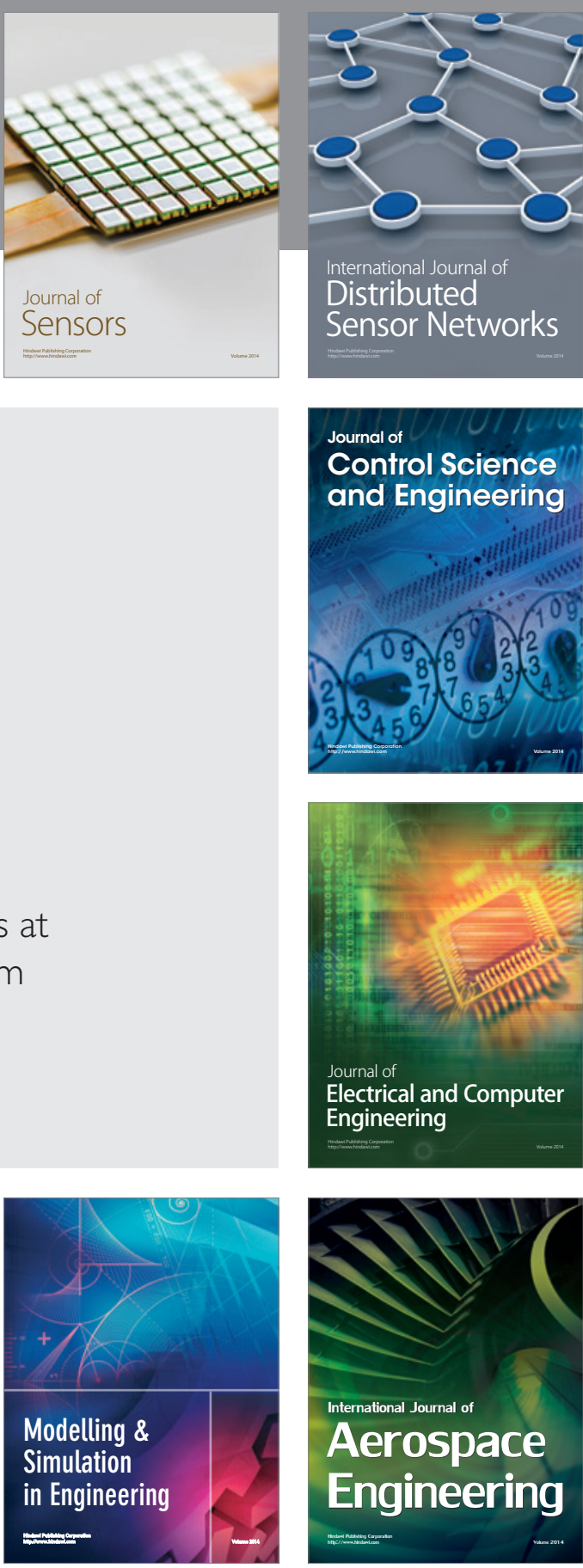

International Journal of

Distributed

Sensor Networks

Journal of

Control Science

and Engineering
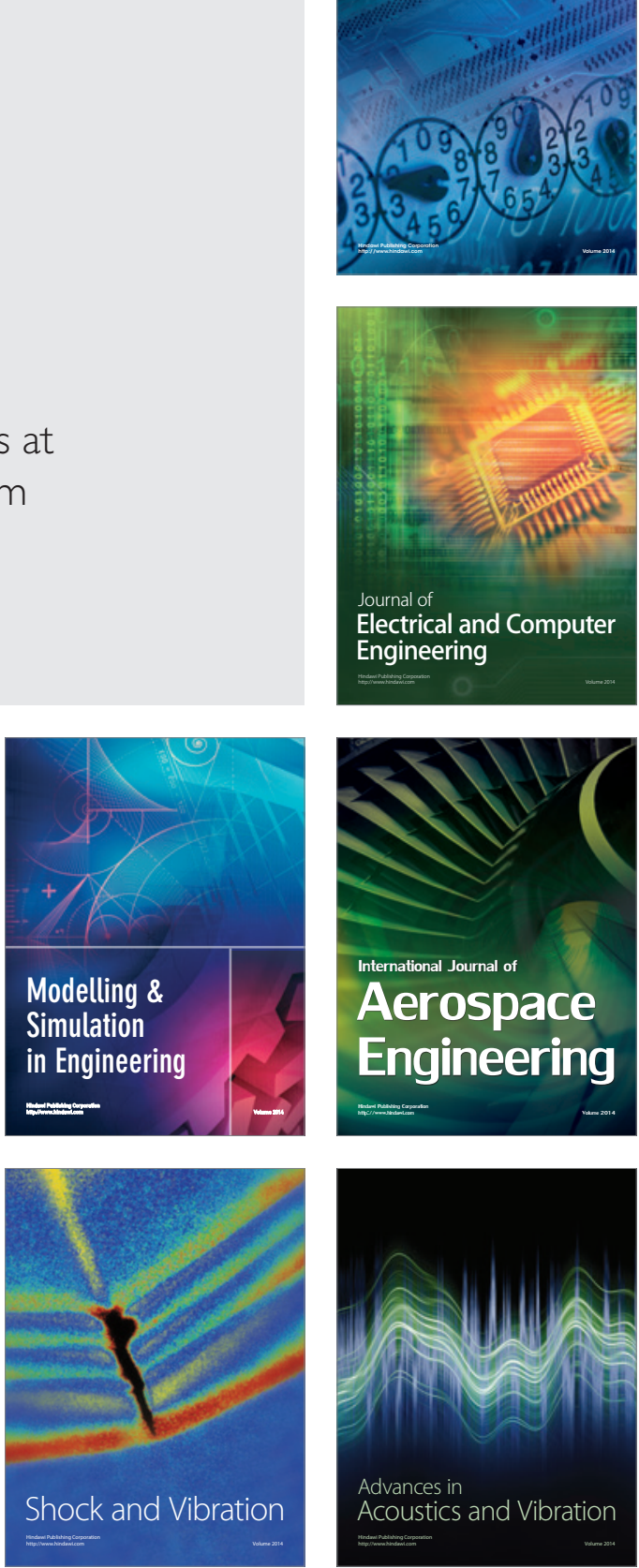